\title{
Decomposition of seismic signals via time-frequency representations
}

Tom Tobback *, Philippe Steeghs, Guy G. Drijkoningen and Jacob T. Fokkema, Section of Applied Geophysics, Centre for Technical Geoscience, Delft University of Technology, the Netherlands

\section{Summary}

In this paper we discuss the use of a time-frequency representation, the Wigner distribution, for the decomposition and characterization of seismic signals. The advantage of the Wigner distribution over other representations, such as the wavelet and sliding window Fourier transform, is its sharp localization properties in the time-frequency plane. However, the Wigner distribution is a not a linear transformation. This non-linearity complicates the use of the Wigner distribution for time-frequency filtering and decomposition. We present an optimization method for the reconstruction of a time signal from its Wigner distribution. The reconstruction technique enables a decomposition of a signal into its time-frequency components, where the reconstructed components are stripped off from the signal one by one. We illustrate the method with a real data example. We also show how the decomposition can be used for suppression and enhancement of events in the time-frequency plane.

\section{Introduction}

In joint time-frequency analysis the time and frequency domain representations of a signal are combined into a time-frequency energy density function. This timefrequency representation can be used to study change of frequency content of a signal with time. There are a number of possible time-frequency representations of which the wavelet (packet) transform and sliding window Fourier transform are the most widely used (see e.g. Chakraborty and Okaya 1995). Our ultimate goal is to characterize the seismic response of the subsurface in terms of its time-varying frequency content (Steeghs and Drijkoningen 1995). In the wavelet transform and windowed Fourier transform representation, time-frequency resolution directly depends on the shape and size of the analysis window or wavelet. A very short time window results in excessive smoothing in frequency and vice versa. As a result of this trade-off between window size and resolution, information is lost or blurred in the timefrequency representations. To circumvent this problem other joint time-frequency representations with better time-frequency localization properties can be used. A time-frequency representation that is optimal with respect to time-frequency localization is the Wigner distribution. The (smoothed) Wigner distribution has been employed in a number of signal characterization, detection and decomposition problems; a comprehensive overview can found in Cohen (1995). A drawback in the application of a time-frequency representation of the Wigner form for time-frequency decomposition and filtering is its non-linearty. This implies that we cannot simply apply a window to the Wigner distribution of a signal and do an inverse transformation, as the windowed part is not necessarily a valid Wigner distribution. In this paper we show how the decomposition of a signal into its time-frequency components can be carried out with an optimization procedure. We discuss two applications of time-frequency signal decomposition. First we show that a hierarchical decomposition of a seismic trace results in the observation that the signal can be characterized by very few and fairly simple time-frequency components. The time extent of these components is almost coincident with seismic facies units. This last observation could be an important step towards a better understanding of the origins and a more quantitative interpretation of seismic reflection patterns. Another application is to use the decomposition for the suppression or enhancement of events in the time-frequency plane.

\section{Theory}

The Wigner distribution is based on the local autocorrelation function $R\{u, u\}(t, \tau)$ of the time signal $u(t)$. This instantaneous auto-correlation is given by

$$
R\{u, u\}(t, \tau)=u(t+\tau / 2) u^{*}(t \Leftrightarrow \tau / 2)
$$

where $\tau$ is the time lag variable and the asterisk denotes complex conjugation. The Wigner distribution, $W\{u, u\}(t, f)$, is the Fourier transform of this autocorrelation:

$$
W\{u, u\}(t, f)=\int_{\tau \in \mathbb{R}} \exp (\Leftrightarrow \dot{j} 2 \pi f \tau) R\{u, u\}(t, \tau) \mathrm{d} \tau .
$$

If a signal is multi-component, i.e. the energy is not well conentrated in the time-frequency plane, cross-terms arise in the Wigner distribution at locations in the $t \Leftrightarrow f$ plane that do not correspond to what is expected (Fig.1). If a signal $u(t)$ is two-component, then

$$
\begin{gathered}
u(t)=c_{1} u_{1}(t)+c_{2} u_{2}(t) \Leftrightarrow \\
W\{u, u\}(t, f)=\left|c_{1}\right|^{2} W\left\{u_{1}, u_{1}\right\}(t, f)+ \\
\left|c_{2}\right|^{2} W\left\{u_{2}, u_{2}\right\}(t, f)+ \\
2 \operatorname{Re}\left\{c_{1} c_{2} W\left\{u_{1}, u_{2}\right\}(t, f)\right\},
\end{gathered}
$$

where $W\left\{u_{2}, u_{1}\right\}(t, f)$ is the cross-Wigner distribution of $u_{1}(t)$ and $u_{2}(t)$ :

$$
\begin{aligned}
W\left\{u_{1}, u_{2}\right\}(t, f)= & \int_{\tau \in \mathbb{R}} \exp (\Leftrightarrow j 2 \pi f \tau) \\
& u_{1}(t+\tau / 2) u_{2}^{*}(t \Leftrightarrow \tau / 2) \mathrm{d} \tau .
\end{aligned}
$$



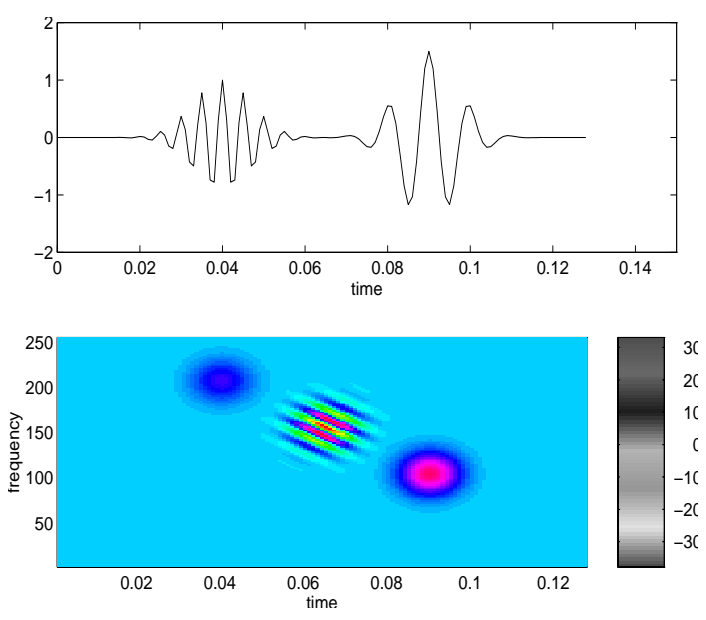

Figure 1: Wigner distribution of a two component signal

This last relation between $t \Leftrightarrow f$ components is important, because it shows that the Wigner distribution of a multicomponent signal contains the Wigner distributions of the individual components. Consequently, if we can separate components in the $t \Leftrightarrow f$ domain, we can decompose a time signal into its time-frequency components by signal synthesis from the Wigner distribution.

\section{Reconstruction}

The Wigner distribution is derived from a one dimensional time signal, so it is clear that its two dimensions $t \Leftrightarrow f$ are not independent. This implies that not any time-frequency function, $\tilde{W}(t, f)$, is a valid Wigner distribution. The Wigner distribution is filtered by multiplying it with a $t \Leftrightarrow f$ mask, $h(t, f)$,

$$
\tilde{W}\{u, u\}(t, f)=h(t, f) \cdot W\{u, u\}(t, f)
$$

To synthesize a time signal from this filtered Wigner distribution we search for a valid Wigner distribution, $W\{x, x\}(t, f)$, that is as near as possible to the masked Wigner distribution, $\tilde{W}(t, f)$. If we have a filtered distribution that is once again a valid Wigner disitribution, we can reconstruct the desired time signal, $x(t)$, out of this $W\{x, x\}(t, f)$. This reconstruction can be formulated as an optimization problem, which results in an orthogonal projection (Hlawatsch and Krattenthaler 1992). We minimize the approximation error; the energetic difference between the filtered $\tilde{W}(t, f)$ and a valid Wigner distribution $W\{x, x\}(t, f)$. This error is minimized over a space $S$ containing all possible time signals $x(t)$ :

$$
\begin{aligned}
& \epsilon_{x}^{2}=\|\tilde{W}(t, f) \Leftrightarrow W\{x, x\}(t, f)\|^{2} \Leftrightarrow \min _{x \in S} \\
& \text { with , } \tilde{W}(t, f) \in \Omega \\
& \text { and , } W\{x, x\}(t, f) \in \Omega_{W},
\end{aligned}
$$

where $\Omega$ is the space of all time-frequency distributions and $\Omega_{W}$ is the space of all Wigner distributions. The solution of this minimization is given by an eigenvalue problem. The result is the function $x(t)$, whose Wigner distribution, $W\{x, x\}(t, f)$, is the closest to $\tilde{W}(t, f)$ (Boudreaux-Bartels and Parks 1986).

\section{Filtering in the $t \Leftrightarrow f$ domain}

To isolate the components in the $t \Leftrightarrow f$ domain, we use rectangular boxcar filters. The problem is that the Wigner distribution of a component extends over a large area in the $t \Leftrightarrow f$ domain, as any bounded signal in time has an infinite frequency spectrum and a time-limited signal has an infinite duration. As a result, we can never isolate an entire component in the Wigner domain with a bounded filter. However, the formulation of the reconstruction problem as an orthogonal projection minimizes the artifacts that are caused by the truncation. We have found that the shape of the masking function $h(t, f)$ has little effect on the results and that the reconstruction algorithm remains stable for a wide variety of window sizes and shapes.

\section{Hierarchical decomposition}

The reconstruction technique enables a decomposition of the signal into its time-frequency components. If we filter a component and by accident include some cross-term energy, the algorithm also reconstructs the unwanted component to some extent. This could cause serious overlap if we would isolate every component from the original Wigner distribution. Therefore we substract each reconstructed component from the signal and then calculate a new Wigner distribution. Another motive for a hierarchical decomposition is the presence of lower amplitude events. Theoretically, the Wigner distribution is a timefrequency representation with infinite $t \Leftrightarrow f$ resolution. Therefore, even very weak signal-components are present in the Wigner distribution. Nevertheless, they often remain undetected, because their amplitude is small compared to the strong components and their cross-terms. The weaker components can be detected if we first subtract the the stronger ones from the signal and then calculate a new Wigner distribution. We decompose the signal into its $t \Leftrightarrow f$ components according to the following scheme. We recall that $u(t)$ is the original signal and $x_{i}(t), i=1,2, \ldots$, is the $i^{t h}$ reconstructed component. The signal $y_{i}(t), i=1,2, \ldots$, is the remaining signal after subtraction of the $i^{\text {th }}$ component. 


$$
\begin{aligned}
& \frac{u(t) \Leftrightarrow W_{0}(t, f)}{\downarrow} \\
& x_{1}(t) \\
& \frac{\downarrow}{y_{1}(t)=u(t) \Leftrightarrow x_{1}(t) \Leftrightarrow W_{1}(t, f)} \\
& \begin{array}{c}
x_{2}(t) \\
\downarrow \\
y_{2}(t)=y_{1}(t) \Leftrightarrow x_{2}(t) \Leftrightarrow W_{2}(t, f)
\end{array} \\
& \begin{array}{c}
x_{N}(t) \\
\downarrow \\
y_{N}(t)=y_{N-1}(t) \Leftrightarrow x_{N}(t)
\end{array}
\end{aligned}
$$

\section{Decomposition of real data}

An illustration of the decomposition is given in Figure 2. The upper part of Fig.(2) shows trace from a marine seismic data set and its Wigner distribution. Note how well the energy is localized in distinct regions of the timefrequency plane. We isolated a $t \Leftrightarrow f$ component in the Wigner distribution and subtracted this component from the trace. In the next step a new Wigner distribution is calculated of the signal minus the first component. Then another component is isolated and subtracted from the signal. This procedure continues until there is no localized energy left in the remaining signal. The maximum amplitudes of the components are shown in Figure 3. The $10^{t h}$ component is much smaller than the $9^{t h}$. This discontinuity is a result of the reconstrution method. A component cannot be entirely subtracted from the Wigner distribution. The energy that is spread out over the masked Wigner distribution introduces noise into the decomposition scheme. We can conclude we have succesfully isolated nine components in this trace. The nature of the remaining energy is uncertain, as it could be noise that was introduced by the decomposition scheme.

\section{Suppression and enhancement of events}

A stratified model (Fig.4) with alternating velocities will have a frequency response that shows strong tuning around the frequencies

$$
f=(2 k \Leftrightarrow 1) \frac{c}{4 d} \text { with } k=1,2, \ldots
$$

In a more realistic model, there will be a certain distribution around an average thickness $d$. It is interesting to see how the $t \Leftrightarrow f$ distribution get more complicated, when the thicknesses are perturbed. Figure 3 shows the Wigner distributions of traces from synthetical geological sequences with a Gaussian thickness distribution with an average of $10 \mathrm{~m}$ and standard deviations $\left(\sigma_{d}\right)$ of $1 \mathrm{~m}, 2 \mathrm{~m}$
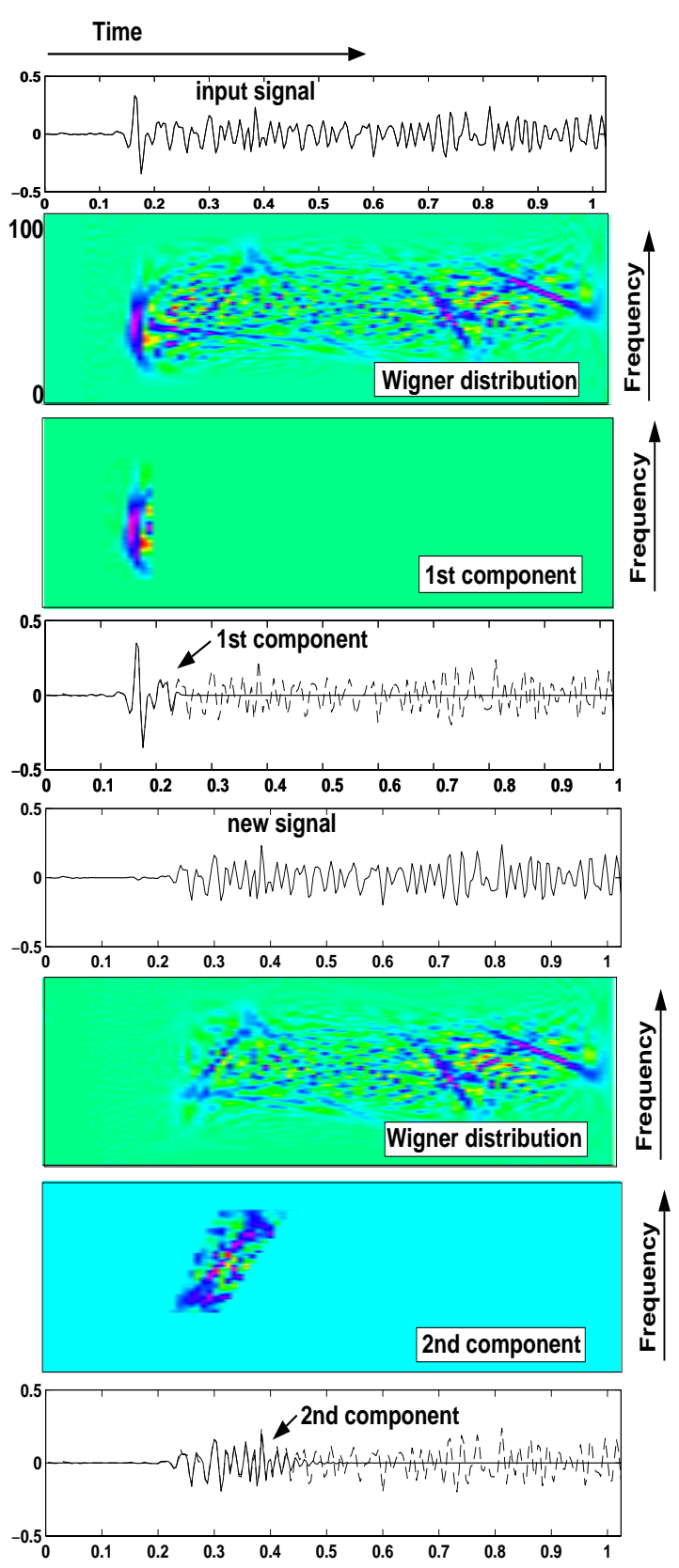

Figure 2: Hierarchical $t \Leftrightarrow f$ decomposition

and $3 \mathrm{~m}$. An application of the decomposition method is illustrated in Figure 5. The model consists of two stratified units of alternating layers with a contrast of $200 \mathrm{~m} / \mathrm{s}$ between the layers. The mean thicknesses are $5 \mathrm{~m}$ and $3 \mathrm{~m}$ and the standard deviations are $1 \mathrm{~m}$ and $0.3 \mathrm{~m}$. In the Wigner distribution we mainly see the strong reflection, the other events are weaker and nearly invisible. Here 


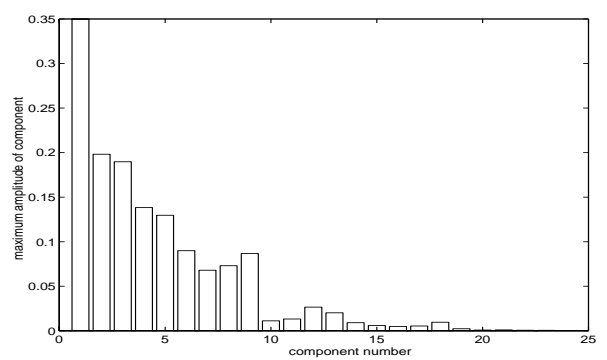

Figure 3: Maximum amplitude of the isolated components
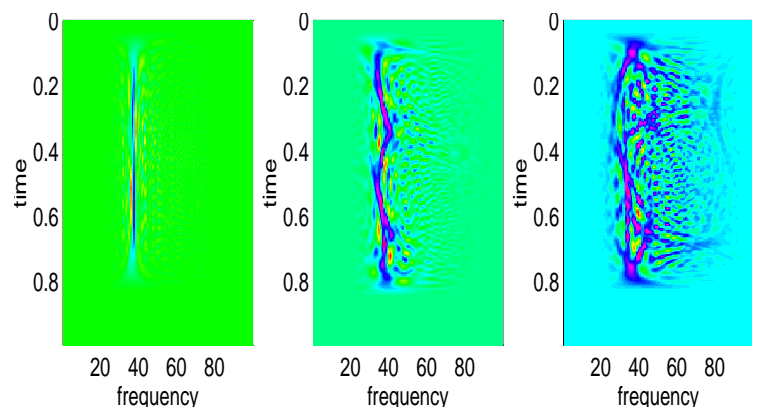

Figure 4: Wigner distributions of models with $d=10(\mathrm{~m})$ and $\sigma_{d}$ of of $1 \mathrm{~m}, 2 \mathrm{~m}$ and $3 \mathrm{~m}$

we can use the decomposition method to substract the strong reflection and enhance the low amplitude information. In the new Wigner distribution, the time-frequency characterisitics sequences becomes very clear and the nature of the stratification can be deduced from the $t \Leftrightarrow f$ representation.

\section{Discussion and conclusions}

We have implemented a decomposition technique to split up a signal into time-frequency components via its Wigner distribution. The method has the advantage over wavelet and Fourier transform based methods because of the sharp and unbiased localization of energy in the Wigner distribution. At present, the decomposition is made rather arbitrarily, as it is not based on a physical model of the seismic signal in the time-frequency plane. However, we observe strong localization of energy in the timefrequency plane. The time-extent of these components generally coincides with distinct seismic facies. The ability to isolate and synthesize these components opens the possibility of further processing and interpretation on isolated seismic facies units. Velocity analysis, time-variant deconvolution and filtering techniques could benefit from such a facies based decomposition. An application of this decomposition technique that was not discussed here is the suppression events that overlap desired signal components in both time and frequency, such as ground-roll or reverberation. A next step is to obtain a better physical
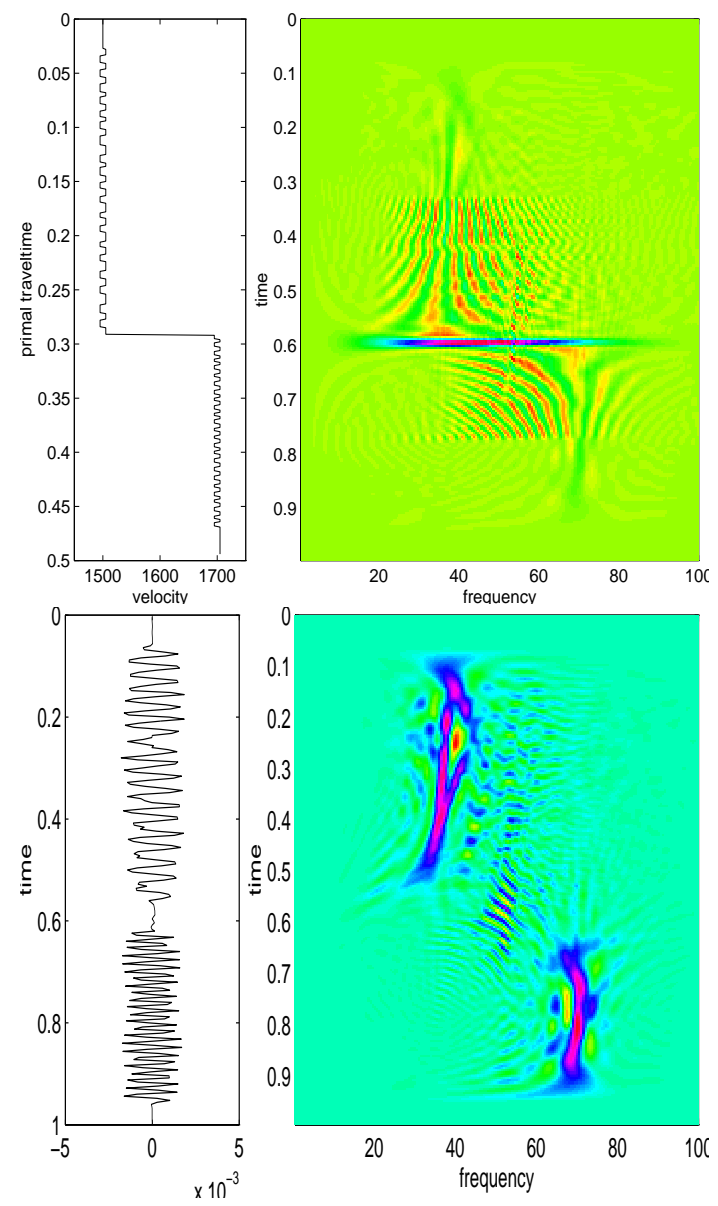

Figure 5: Stratified model and Wigner distribution of the seismic response, (top), filtered signal and Wigner distribution of the $t \Leftrightarrow f$ filtered signal (bottom)

basis for the time-frequency decomposition, for instance in relation to frequency-tuning in stratified media.

\section{References}

Chakraborty, A., Okaya, D.A., 1995 Frequency-time decomposition of seismic data using wavelet based methods, Geophysics, vol.60(6), pp. 1906-1916.

Cohen, L., 1995, Time-Frequency Analysis, Prentice Hall PTR, Englewood Cliffs, New Jersey.

Hlawatsch, F. and Krattenthaler, W., 1992, Bilinear signal synthesis, IEEE Trans. on SP, vol.40(2), pp. 352-363.

Boudreaux-Bartels, G.F., and Parks, T, 1986 Timevarying filtering and signal estimation using Wigner distribution synthesis techniques, IEEE Transactions on ASSP, vol.34(3), pp. 442-451.

Steeghs, T.P.H. and Drijkoningen, G.G., 1995, Timefrequency analysis of seismic sequences, Expanded Abstracts, 65th Annual Meeting of the SEG, Houston. 